\title{
Anharmonic Lattice Vibrations and the Temperature Shift of Raman Spectral Lines*
}

\author{
Christovam Mendonça ${ }^{a}$ and Said R. Rabbani ${ }^{b}$ \\ a Departamento de Física, Universidade Federal de São Carlos, São Carlos, SP, \\ CEP 13565-905, Brazil \\ b Instituto de Física, Universidade de São Paulo, Caixa Postal 66318, São Paulo, SP, \\ CEP 05389-970, Brazil
}

Z. Naturforsch. 51a, 716-720 (1996); received November 20, 1995

\begin{abstract}
The temperature dependence of the average lattice mode frequency of a molecule undergoing librational motion in a quasi-harmonic potential has been calculated with the purpose of finding an explicit relationship between the observed shift of Raman spectral lines with temperature and the anharmonic term in the rotational potential. Calculations were carried out both for uncoupled and coupled oscillators. The equations obtained with this model provide good fittings for solid $\mathrm{Cl}_{2}$ and benzene data. This result can be applied to the analysis of the temperature dependence of NQR frequencies in molecular crystals
\end{abstract}

Key words: Vibrational Spectroscopy, Raman Spectra, Anharmonic Vibrations, NQR.

\section{Introduction}

Raman studies of molecular crystals are of interest for nuclear quadrupole resonance (NQR) spectroscopy because molecular and lattice vibrations cause high frequency modulations of the quadrupole interaction, resulting in changes of NQR frequencies with temperature and relaxation as well.

In the rigid molecule model proposed by Bayer [1], only harmonic librational motions of isolated molecules, with frequencies much higher than the NQR frequency, contribute to the averaging of the quadrupole interaction. So, each molecule oscillates with two constant frequencies, which reduce to a single one if the molecule possesses axial symmetry, Bayer's equation can generally be fitted quite well to low temperature data, but increasing disagreement with experimental values appears when the fitted curve is extrapolated to higher temperatures. Attempts to extract librational frequencies as regression parameters also may be unsuccessful, yielding values not coinciding with those measured in Raman spectroscopy. Bayer's model does not take into account the coupling due to intermolecular bonding and the decrease of librational frequencies with increasing temperature.

\footnotetext{
* Presented at the XIIIth International Symposium on Nuclear Quadrupole Interactions, Providence, Rhode Island, USA, July 23-28, 1995.

Reprint requests to Prof. Christovam Mendonça.
}

This effect is currently observed in Raman spectra and claimed to be a consequence of vibrational anharmonicity and thermal expansion. A linear relationship is often assumed, and Brown [2] showed that Bayer's model could be improved by introducing the empirical temperature dependence of the librational frequencies $v_{l}=v_{l_{0}}(1-g T)$. The observation of Raman spectra taken over wide temperature ranges, however, such as those made by Cahill and Leroi [3] in solid $\mathrm{Cl}_{2}$ and by Ito and Shigeoka [4] in benzene, reveals an obvious nonlinear behaviour. In the attempt to disclose anharmonic effects within NQR frequency versus temperature curves, Gillies and Brown [5] adjusted Bayer's model to molecules undergoing librational in a pure quartic potential. They showed that the existence of a predominant motion of this type would lead to a nonmonotonic rate of decrease of frequency with temperature. Since this is not observed in molecular crystals showing no phase transitions, they concluded that strong anharmonicities do not exist.

In this work we propose a different approach, in which lattice frequencies become temperature dependent as a result of slightly distorted harmonic potentials.

\section{Single Anharmonic Oscillator}

We consider a molecule undergoing torsional oscillations about one of the principal axes of inertia. If $\theta$ 
is the angular displacement from its equilibrium orientation, the restoring potential is assumed to have the form

$$
V(\theta)=(1 / 2) I \omega_{0}^{2} \theta^{2}-\lambda \theta^{4},
$$

where $I$ is the molecular moment of inertia about the oscillation axis and $\lambda$ is the anharmonic coefficient, obeying the condition

$$
\lambda\left\langle\theta^{2}\right\rangle \ll I \omega_{0}^{2} / 2,
$$

so that we can apply first order perturbation theory to calculate energy eigenvalues. The reasons for the choice of a quartic term as the only distortion of the harmonic potential are: (1) the cubic term does not contribute to a perturbation correction in first order and (2) unsymmetrical restoring forces are not expected in rotational oscillations. We shall see that a negative quartic term is consistent with negative frequency shifts. Let $|n\rangle$ be the simple harmonic oscillator eigenstate labeled by the quantum number $n$, then the energies will be

$$
\begin{aligned}
& E_{n}=\hbar \omega_{0}(n+1 / 2)-\lambda\left\langle n\left|\theta^{4}\right| n\right\rangle, \\
&(n=0,1, \ldots) .
\end{aligned}
$$

The calculation of the diagonal element $\theta_{n n}^{4}$ is straightforward, the result being

$E_{n}=\hbar \omega_{0}(n+1 / 2)-\left(3 \hbar^{2} / 2 I^{2} \omega_{0}^{2}\right) \lambda\left(n^{2}+n+1 / 2\right)$.

It is convenient to introduce the quantity $\Omega$ :

$$
\Omega=\left(3 \hbar / 2 I^{2} \omega_{0}^{2}\right) \lambda,
$$

Then (4) becomes

$$
E_{n}=\hbar \omega_{0}(n+1 / 2)-\hbar \Omega(n+1 / 2)^{2}-\hbar(\Omega / 4) .
$$

The frequency shifts occurring in Stokes mode Raman processes are given by $\left(E_{n+1}-E_{n}\right) / \hbar$, so

$$
\omega_{n}^{s}=\omega_{0}-2 \Omega(n+1) .
$$

Equations (4) and (6) show that anharmonicity results in unequally separated energy levels, leading to the appearance of additional lines of different frequencies as the temperature is raised and upper levels become populated. This explains the line broadening. The central frequency is now evaluated as the ensemble average of the frequencies $\omega_{n}^{s}$ :

$$
\left\langle\omega^{s}\right\rangle=\omega_{0}-2 \Omega \sum_{n}(n+1) e^{-x(n+1 / 2)} / \sum_{n} e^{-x(n+1 / 2)},
$$

where $x=\hbar \omega_{0} / k T$, and the unperturbed partition function is considered since we are keeping just first order corrections in $\Omega$, i.e., $\lambda$. The temperature dependence of $\omega^{s}$ is easily calculated as

$$
\omega(T)=\omega_{0}-\Omega\left[\operatorname{coth}\left(\hbar \omega_{0} / 2 k T\right)+1\right],
$$

where the superscript $S$ was omitted, since the same expression is obtained in the anti-Stokes mode calculation. For very low temperatures, the line frequency is

$$
\omega(0)=\omega_{0}-2 \Omega,
$$

so that even at absolute zero the anharmonic effect can be noted. For high temperatures, $k T \widetilde{>} \hbar \omega_{0}$, the frequency shift shows that asymptotic linear feature assumed in Brown's correction:

$\omega_{\infty}(T)=\left(\omega_{0}-\Omega\right)(1-g T) \neq \omega(0)(1-g T)$,

where $g \cong\left(2 \Omega \mathrm{k} / \hbar \omega_{0}^{2}\right)$.

\section{Two Coupled Anharmonic Oscillators}

Let two identical molecules, undergoing anharmonic oscillations under restoring torques derived from potentials given by (1), be connected by harmonic couplings. Let $\theta_{1}$ and $\theta_{2}$ be the instantaneous angular displacements. The total Hamiltonian is then written as

$$
\begin{aligned}
H= & T_{1}+T_{2}+(1 / 2) I \omega_{0}^{2}\left(\theta_{1}^{2}+\theta_{2}^{2}\right) \\
& +(1 / 2) I K_{1}^{2}\left(\theta_{1}+\theta_{2}\right)^{2} \\
& +(1 / 2) I K_{2}^{2}\left(\theta_{1}-\theta_{2}\right)^{2}-\lambda\left(\theta_{1}^{4}+\theta_{2}^{4}\right),
\end{aligned}
$$

where $T_{1}$ and $T_{2}$ are the kinetic energy operators and $K_{1}$ and $K_{2}$ are frequencies associated to the symmetric and antisymmetric oscillation couplings. Introducing the normal coordinates

$$
\begin{aligned}
& q_{1}=\sqrt{I / 2}\left(\theta_{1}+\theta_{2}\right), \\
& q_{2}=\sqrt{I / 2}\left(-\theta_{1}+\theta_{2}\right),
\end{aligned}
$$

the Hamiltonian is transformed into

$$
\begin{aligned}
H= & -\left(\hbar^{2} / 2\right)\left[\partial_{1}^{2}+\partial_{2}^{2}\right]+(1 / 2) \omega_{10}^{2} q_{1}^{2} \\
& +(1 / 2) \omega_{20}^{2} q_{2}^{2}-\left(\lambda / 2 I^{2}\right) q_{1}^{4}-\left(\lambda / 2 I^{2}\right) q_{2}^{4} \\
& -\left(3 \lambda / I^{2}\right) q_{1}^{2} q_{2}^{2},
\end{aligned}
$$

where $\omega_{10}^{2}=\omega_{0}^{2}+2 K_{1}^{2}, \omega_{20}^{2}=\omega_{0}^{2}+2 K_{2}^{2}, \partial_{k}^{2} \equiv \partial^{2} /$ $\partial q_{k}^{2}(k=1,2)$, and is not uncoupled into two independent oscillators because the $q_{1}^{2} q_{2}^{2}$ term originates in the anharmonicity, not in a mechanical coupling. Treating the terms with $\lambda$ as perturbations, in a procedure analogous to that followed in the preceding sec- 
tion, we obtain the energies

$$
\begin{aligned}
E\left(n_{1}, n_{2}\right)= & \hbar \omega_{10}\left(n_{1}+1 / 2\right)+\hbar \omega_{20}\left(n_{2}+1 / 2\right) \\
& -(1 / 2) \hbar \Omega_{1}\left(n_{1}^{2}+n_{1}+1 / 2\right) \\
& -(1 / 2) \hbar \Omega_{2}\left(n_{2}^{2}+n_{2}+1 / 2\right) \\
& -2 \hbar\left(\Omega_{1} \Omega_{2}\right)^{1 / 2}\left(n_{1}+1 / 2\right)\left(n_{2}+1 / 2\right), \\
& \left(n_{1}, n_{2}=0,1 \ldots\right), \quad(15)
\end{aligned}
$$

where

$$
\Omega_{1,2}=3 \hbar \lambda / 2 I^{2} \omega_{1,2}^{2} .
$$

The transition frequencies $\omega_{S}$ and $\omega_{A}$ for the symmetric and antisymmetric modes are

$\omega_{S}=\omega_{10}-\Omega_{1}\left(n_{1}+1\right)-2\left(\Omega_{1} \Omega_{2}\right)^{1 / 2}\left(n_{2}+1 / 2\right)$

and

$\omega_{A}=\omega_{20}-\Omega_{2}\left(n_{2}+1\right)-2\left(\Omega_{1} \Omega_{2}\right)^{1 / 2}\left(n_{1}+1 / 2\right)$.

The central frequencies, calculated through the ensemble averages of $\omega_{S}$ and $\omega_{A}$, are given by

$$
\begin{aligned}
\omega_{1}(T)= & \omega_{10}-(1 / 2) \Omega_{1}\left[\operatorname{coth}\left(\hbar \omega_{10} / 2 k T\right)+1\right] \\
& -\left(\Omega_{1} \Omega_{2}\right)^{1 / 2} \operatorname{coth}\left(\hbar \omega_{20} / 2 k T\right)
\end{aligned}
$$

and

$$
\begin{aligned}
\omega_{2}(T)= & \omega_{20}-(1 / 2) \Omega_{2}\left[\operatorname{coth}\left(\hbar \omega_{20} / 2 k T\right)+1\right] \\
& -\left(\Omega_{1} \Omega_{2}\right)^{1 / 2} \operatorname{coth}\left(\hbar \omega_{10} / 2 k T\right) . \quad(18 \mathrm{~b})
\end{aligned}
$$

For temperatures close to the absolute zero the lines have the frequencies

$$
\omega_{1}(0)=\omega_{10}-\Omega_{1}-\left(\Omega_{1} \Omega_{2}\right)^{1 / 2}
$$

and

$$
\omega_{2}(0)=\omega_{20}-\Omega_{2}-\left(\Omega_{1} \Omega_{2}\right)^{1 / 2},
$$

\section{Fitting to Experimental Data}

\section{a. Solid Chlorine}

Solid chlorine has the orthorhombic crystal structure, with four $\mathrm{Cl}_{2}$ molecules in the face centered unit cell and two molecules per primitive cell, arranged in layers parallel to the $b c$ crystallographic plane. The crystal presents four zero-wave vector librational modes $\left(A_{g}+B_{1 g}+B_{2 g}+B_{3 g}\right)$, all of them expected to be Raman active. Raman studies of this crystal have been made by several authors $[3,6-8]$, with partial agreement between reported frequencies and line assignments. Combined analyses of NQR and Raman data were performed by McEnnan and Schempp [9], and Martin [10].
We elected the frequencies reported by Cahill and Leroi [3] because they carried out measurements over a wide temperature range with sufficient experimental points to test our model. However, only three frequencies were detected and independently confirmed by other authors, out of the four librational frequencies expected.

The absence of a fourth line causes disagreement about the assignments of the frequencies to the four symmetry coordinates, and we refer to McEnnan and Schempp's paper for this dispute [9]. Let us just mention that Anderson and Sun [6] suggested that an intense and broad line near $100 \mathrm{~cm}^{-1}$ corresponds to the accidental overlap of the $A_{g}$ (inter-layer) and $B_{2 g}$ (intra-layer) modes, which is supported by the intermolecular potential calculations by Dumas et al. [8]. Cahill and Leroi [3] suggested that the missing line stays near $60 \mathrm{~cm}^{-1}$, on the basis of the relative frequencies of the four librational modes observed in solid bromine. This hypothesis is supported by nuclear quadrupole resonance frequency data, as verified by Obermyer and Jones [11], and is the subject of a paper of ours [12].

The Raman data for solid chlorine are listed in Table 1, where we included the data suggested by

\begin{tabular}{|c|c|c|c|c|c|}
\hline \multicolumn{3}{|c|}{ Fitting to eq. (8) } & \multicolumn{3}{|c|}{ Fitting to eq. (18) } \\
\hline $\bar{v}_{0}$ & $\Omega$ & $v(0)$ & $\bar{v}_{0}$ & $\Omega$ & $v(0)$ \\
\hline 63.8 & 1.8 & 60 & 63.5 & 1.4 & 60 \\
\hline 92 & 4.4 & 83 & 88.1 & 2.9 & 83.2 \\
\hline 122 & 11 & 100 & 119 & 7.7 & 99.7 \\
\hline 184 & 33 & 118 & 148 & 17.6 & 119 \\
\hline
\end{tabular}
Cahill and Leroi for the missing line. Equations (8) and (18), fitted to the values listed in Table 1, lead to the evaluation of the force constant frequency $\omega_{0}$, whose measure in $\mathrm{cm}^{-1}$ is represented as $\bar{v}_{0}$, and the

Table 1. $\mathrm{Cl}_{2}$ Raman Libration Frequencies in $\mathrm{cm}^{-1}$ at various temperatures, [3]. The values in the first line were not experimentally observed.

\begin{tabular}{ccccl}
\hline $15 \mathrm{~K}$ & $68 \mathrm{~K}$ & $84 \mathrm{~K}$ & $118 \mathrm{~K}$ & $170 \mathrm{~K}$ \\
\hline 60 & 59 & 58.5 & 57 & 55 \\
83 & 81.5 & 81 & 79 & 75.5 \\
100 & 97.5 & 96 & 93.5 & 87 \\
118.5 & 115.5 & 114 & 109.5 & 99.5 \\
\hline
\end{tabular}

Table 2. Fitting of coupled and uncoupled oscillator equations to $\mathrm{Cl}_{2}$ Raman data listed in Table $1 . \bar{v}_{0}$ and $\Omega$ are in $\mathrm{cm}^{-1}$. 


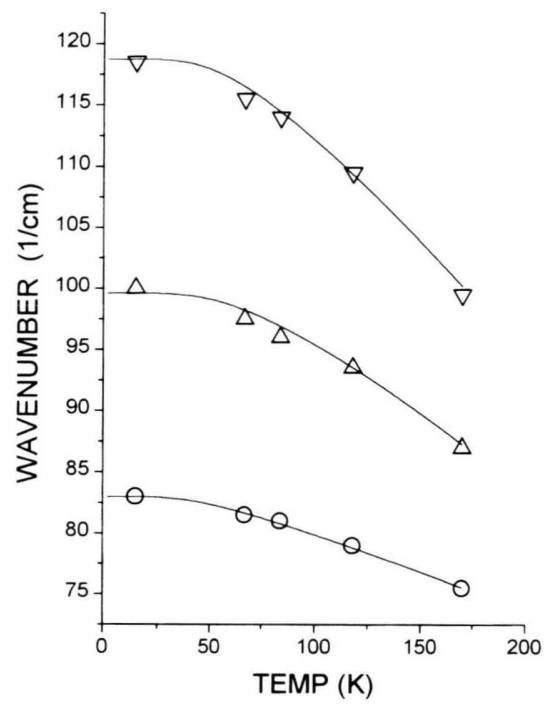

Fig. 1. Solid chlorine librational frequencies and fitting curves, single oscillator model, (8).

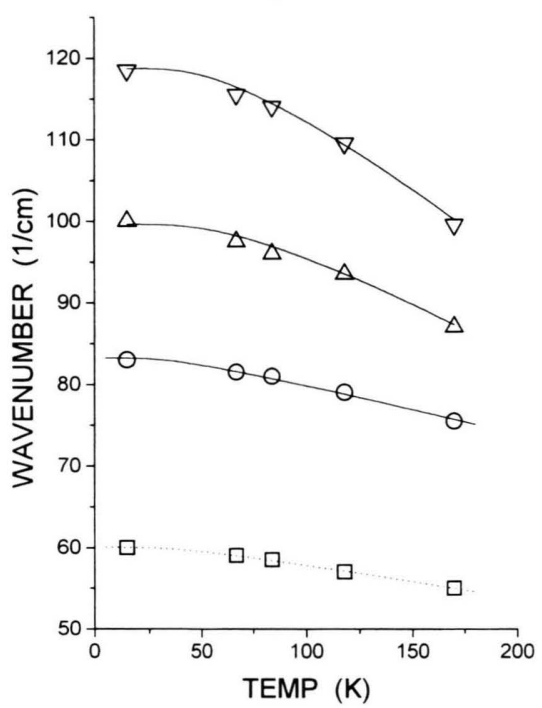

Fig. 2. Solid chlorine data and fitting curves, coupled oscillators model, $(17 \mathrm{a}, \mathrm{b})$. The dotted curve is a fitting for the suggested [3] values of the missing $\mathrm{Cl}_{2}$ line, presumed to be coupled to the $83 \mathrm{~cm}^{-1}$ mode.

anharmonic contribution $\Omega$, responsible for the temperature shift. The results are summarized in Table 2, and the single oscillator model can be compared to the more realistic picture of two coupled oscillators. The calculated absolute zero frequencies are also tabulated. The fitted curves for both cases are shown in Figs. 1 and 2; they are essentially coincident.
It can be seen that, although the single oscillator model provides a satisfactory fitting, it reproduces the linear trend at higher temperatures as well as the plateau in the low temperature range, the ratio $\Omega / \bar{v}_{0}$ can hardly be considered small enough to justify the perturbation treatment. This is best achieved in the fitting of (18). The parameters were extracted by simultaneous regression of the band pairs $60-83 \mathrm{~cm}^{-1}$ and $100-118 \mathrm{~cm}^{-1}$.

\section{b. Solid Benzene and Deuterated Benzene}

Raman spectra at temperatures between $4 \mathrm{~K}$ and $273 \mathrm{~K}$ have been studied by Ito and Shigeoka (I \& S) [4] for benzene and benzene- $d_{6}$ crystals. They emphasized the nonlinear frequency decrease, being very small below $77 \mathrm{~K}$ and considerably larger at higher temperatures. The interest in their measurements lies in the possibility of comparing librations of similar molecules with known ratio between moments of inertia. Therefore, for librational motion the ratio between the parameters $\omega_{0}$, related to the harmonic part of the restoring force, for the corresponding bands of the pure and deuterated benzene crystals are expected to be

$\omega_{0}(H) / \omega_{0}(D)=\bar{v}_{0}(H) / \bar{v}_{0}(D)=[I(D) / I(H)]^{1 / 2}$,

while (5) says that the parameters $\Omega$ must be related as

$$
\Omega(H) / \Omega(D)=[I(D) / I(H)],
$$

where $I(H)$ and $I(D)$ are the moments of inertia of the benzene and the benzene- $\mathrm{d}_{6}$ molecules. $I(D) / I(H)$ is equal to 1.21 for any one of the symmetry axes, so the expected values for the ratio between the $\omega$ 's is 1.10 .

Four bands were selected within the two sets of I \& S Raman data. The benzene crystal has 4 molecules in a unit cell, so 12 librational modes exist, and the extension of $(18 \mathrm{a}, \mathrm{b})$ to this condition would lead to a cumbersome expression and a high uncertainty in the frequency assignments, let alone the evaluation of the parameters. The expression for a single oscillator, (8), was then fitted to each band. Figure 3 shows the reported data at four different temperatures and the best fit curves for pure benzene. A similar plot for deuterated benzene is entirely analogous, with frequencies shifted toward lower values. The fitting parameters $\bar{v}_{0}$ and $\Omega$ are listed in Table 3. It can be observed in the two columns on the right that the determined $\bar{v}_{0}$ ratios are close to the expected values, the estimated error being \pm 0.03 , whereas the $\Omega$ ratios, with estimated 


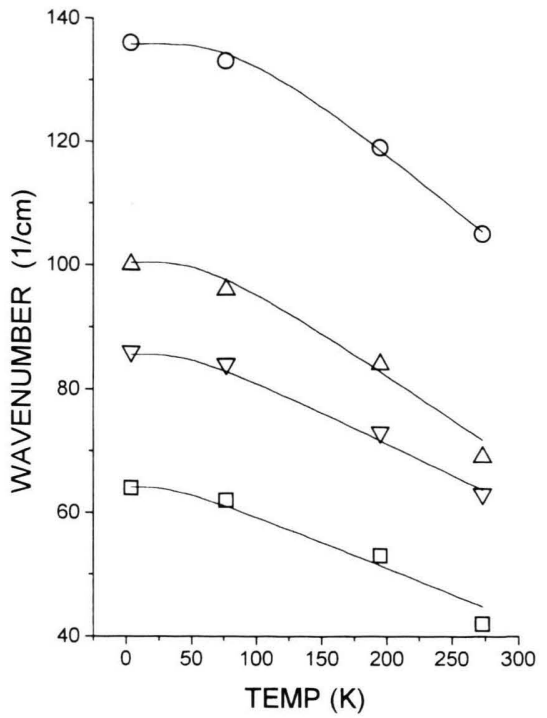

Fig. 3. Solid benzene librational frequencies and fitting curves, single oscillator model.

Table 3. Benzene and Benzene- $\mathrm{d}_{6}$ Libration Frequencies in $\mathrm{cm}^{-1}$ (I\&S [4]) and fitting parameters $\bar{v}_{0}$ and $\Omega$.

\begin{tabular}{lrrrrrrrrr}
\hline $\begin{array}{l}\text { I\&S } \\
\text { label }\end{array}$ & $\begin{array}{l}\mathrm{C}_{6} \mathrm{H}_{6} \\
(4 \mathrm{~K})\end{array}$ & $\bar{v}_{0}$ & $\Omega$ & $\begin{array}{c}\mathrm{C}_{6} \mathrm{D}_{6} \\
(4 \mathrm{~K})\end{array}$ & $\bar{v}_{0}$ & $\Omega$ & $\frac{\bar{v}_{0}(H)}{\bar{v}_{0}(D)}$ & $\frac{\Omega(H)}{\Omega(D)}$ \\
\hline$v_{1}^{l}$ & 64 & 73 & 4.5 & 57 & 64.4 & 3.6 & 1.14 & 1.25 \\
$v_{3}^{l}$ & 86 & 102 & 7.8 & 77 & 88.0 & 5.5 & 1.16 & 1.40 \\
$v_{4}^{l}$ & 100 & 128 & 14.0 & 90 & 109 & 9.4 & 1.17 & 1.47 \\
$v_{6}^{l}$ & 136 & 187 & 25.5 & 123 & 159 & 18 & 1.18 & 1.42 \\
\hline
\end{tabular}

error \pm 0.25 , are in poor agreement with that given by (21), except for the $v_{1}^{l}$ band. This may be due to the uncoupled oscillator approximation. In [4] I\&S pointed out that all the frequencies of the corresponding lines in pure and deuterated benzene have ratios close to 1.10 , which meant that these spectral lines can be assigned to harmonic librational motions. We verified that this is only a coincidence, for the same rela-

[1] H. Bayer, Z. Physik 130, 227 (1951).

[2] R. J. C. Brown, J. Chem. Phys. 32, 116 (1960).

[3] J. E. Cahill and G. E. Leroi, J. Chem. Phys. 51, 4514 (1969).

[4] M. Ito and T. Shigeoka, Spectroch. Acta 22, 1029 (1966).

[5] G. C. Gillies and R. J. C. Brown, J. Magn. Reson. 9, 8 (1973).

[6] A. Anderson and T. S. Sun, Chem. Phys. Lett. 6, 611 (1970).

[7] M. Suzuki, T. Yokoyama, and M. Ito, J. Chem. Phys. 50, 3392 (1969). tionship occurs with our calculated curves fed with best fit parameters in the whole temperature range, despite the anharmonicity effects included.

\section{Discussion}

We have used an extremely simple model of anharmonic lattice vibrations to show that it can explain the general features of the frequency decrease of Raman bands with temperature increase, as observed in simple molecular crystals. The calculated equations shows that anharmonicity cannot be neglected even at absolute zero, and a quasilinear change occurs if temperatures are high enough. It was assumed that an anharmonic term in the rotational potential does not alter the transition selection rules, and no volume expansion was taken into account.

Despite the reasonably good fitting obtained for the calculated expressions to data on chlorine and benzene crystals, and the good agreement between calculated and measured (fitted) ratios of the harmonic and anharmonic force constants for benzene and benzene$\mathrm{d}_{6}$, as listed in Table 3, the relative values of $\omega_{0}$ and $\Omega$ obtained through nonlinear regression may be questioned, for if they are substituted into (6) or (17a, b), the model of a Raman band having a structure of sharp lines will fail: their separation $2 \Omega$ would certainly make them appear as resolved lines. Since this is not the case, we feel there are two possibilities to be investigated: $\omega_{0}$ itself may change with temperature as a result of (i) widening of the potential well due to thermal expansion, and/or (ii) motional averaging of the intermolecular interaction.

\section{Acknowledgement}

C. M. wishes to thank the Departamento de Física Geral, Instituto de Física, Universidade de São Paulo, for the hospitality extended to him during his sabbatical leave.

[8] G. G. Dumas, F. Vounelle, and J.-P. Viennot, Mol. Phys. 28, 1345 (1974).

[9] M. M. McEnnan and E. Schempp, J. Magn. Reson. 11, $28(1973)$.

[10] C. A. Martin, J. Magn. Reson. 64, 1 (1985).

[11] R. T. Obermyer and E. P. Jones, Can. J. Phys. 51, 2300 (1973).

[12] C. Mendonça and S. R. Rabbani, Z. Naturforsch. 51 a, 700 (1996). 